\title{
Der Bauplan der Keimdrüsen ${ }^{1}$. Von \\ Prof. Dr. Alfred Kohn, Prag. \\ Mit Tafel VI und 7 Textabbildungen. \\ (Eingegangen am 27. November 1919.)
}

I.

Wenn man einigen neueren Autoren Glauben schenken dürfte, stehen wir inmitten einer bemerkenswerten Wandlung unserer Anschauungen über die Keimdrüsen.

Diese bestehen bekanntlich aus einem generativen Anteil (Follikel im Ovar, Samenkanälchen im Hoden), in welchem die Keimzellen lagern und reifen, und einem intergenerativen Anteil, den Zwischenzellen, deren Natur und Bedeutung noch nicht genügend aufgeklärt ist.

Fs heißt nun, daß wir den generativen Anteil bisher überschätzt haben. Unbestritten bleibt natürlich seine Bedeutung für die Fortpflanzung; aber alle anderen, den somatischen und psychischen Geschlechtscharakter bestimmenden Wirkungen, sollen nicht, wie wir früher glaubten, von ihm ausgehen, sondern von den $Z$ wischenzellen (Glandula interstitialis oder »Pubertätsdrüse«) ausgelöst werden. Wenn man früher der Ansicht war, daß die Geschlechtslust, die sich besonders lebhaft beim Männchen kundgibt und in der kampffrohen Rivalität der Brunstzeit ihren energischsten Ausdruck findet, mit der periodischen Reifung der Samenzellen in Zusammenhang stehe, so wird dies jetzt als Irrtum hingestellt. Nur die Zwischenzellen allein sollen es sein, die durch innere Sekretion das männliche Wesen, die Sinnenlust und Kampfstimmung hervorrufen; je mehr Zwischenzellen, desto ausgesprochener der Geschlechtscharakter, so daß man zu der seltsamen Folgerung gelangte, daß jene Individuen als die männlichsten erscheinen sollen, deren Hoden nur Zwischenzellen und gar keine Samenkanälchen enthalten.

Zwar ist die Erfahrung mit einer solchen Annahme schwer in Einklang zu bringen; denn die reichlichsten Zwischenzellen findet man in den Hoden von Feten, von Hermaphroditen und in sonșt patho-

1) Nach einem Vortrag, der am 25. Oktober $1918 \mathrm{im}$ "Verein deutscher Ärzte « in Prag gehalten wurde. 
logisch veränderten Hoden, ohne irgendwelche Zeichen gesteigerter Männlichkeit; zwar sind die Zwischenzellen gerade in der Zeit der Geschlechtsruhe am zahlreichsten und in der Begattungszeit am spärlichsten (s. Textabb. 6 und 7 ), aber die Gläubigen der neuen 》 Pubertätslehre lassen sich von Einwänden und Bedenken solcher Art nicht irremachen. Sie haben vielmehr aus ihren Ansehauungen schon weitgehende Schlüsse und praktische Folgerungen gezogen; die Rütsel der Geschlechtsbestimmung, des Hermaphroditismus und der Homosexualität bereiten ihnen keine schwierigkeiten mehr.

Unter solchen Umständen erscheint es dringend geboten, die wissenschaftlichen Grundlagen der Lehre von den Keimdrüsen klarzustellen, und dies will ich, soweit es der Worpholog auf Grund fremder und eigener Untersuchungen vermag, im folgenclen versuchen. Ausgehen wollen wir von der Entwicklungsgeschichte; denn nur sie allein vermag in die verwirrende Mannigfaltigkeit, welche insbesondere die weibliche geschlechtsreife Keimdrüse darbietet, einiges Licht zu bringen.

II.

Die ersten Entwicklungsstufen zeigen bei beiden Geschlechtern ein vollkommen übereinstimmendes Bild, so daß man von einer indifferenten Anlage der Keimdrüsen zu sprechen pflegt. Dieser Bezeichnung liegt offenbar die Vorstellung zugrunde, daß die Keimdrüse - und mit ihr natürlich auch der Embryo - zu dieser Zeit noch nicht geschlechtlich determiniert sei und die endgültige Geschlechtsbestimmung erst später erfolge. Wir stehen aber heute auf dem Standpunkte, dab im allgemeinen schon in der befruchteten Eizelle der künftige Werdegang des Individuums - ungestörte Entwicklung vorausgesetzt - vorgezeichnet sei und somit schon der einzellige Embryo ein ganz' bestimmtes und daher auch geschlechtlich determiniertes Einzelwesen in seiner einfachsten Erscheinungsform clarsteile. So geht z. B. aus dem vom mütterlichen Organismus losgelösten Hühnerei in selbständiger und unbeeinflußter Entwicklung ein Hühnchen hervor, von bestimmter Art, bestimmtem Gefieder und bestimmtem Geschlecht. 'Wenn wir gegenwärtig die Keimdrïsen in einem gewissen Stadium noch nicht zu unterscheiden vermögen, so sind wir gleichwohl überzeugt, daß über das Geschlecht des Embryo schon endgültig entschieden sei. Richtig bleibt aber, daß die beiden Keimdrïsen in ihren frühesten Entwicklungsstufen vollkommen homolog sind und einen übereinstimmenden Bauplan zeigen, wie sich aus der folgenden Darstellung ergeben wird.

Die erste Anlage erscheint in Form der flächenhaften Keimdriise. Es ist dies die bekannte Epithelplatte, die von Waldeyer den Namen "Keimepithel« erhielt und aus umgewandelten Cölomzellen mit eingelagerten Geschlechtszellen besteht. 
Daraus entwickelt sich durch lebhafte Zellvermehrung ein ansehnlicher Zellwulst, der unterhalb der oberflächlichen, platten Zelldecke bald eine gewisse Sonderung in Zellstränge erkennen läßt. Auf diese Weise wird eine Zweiteilung des Organs angebahnt, in eine oberflächliche platte Rindenschicht und eine strangartig angeordnete Markschicht. Man kann dieses zweite Stadium als das der

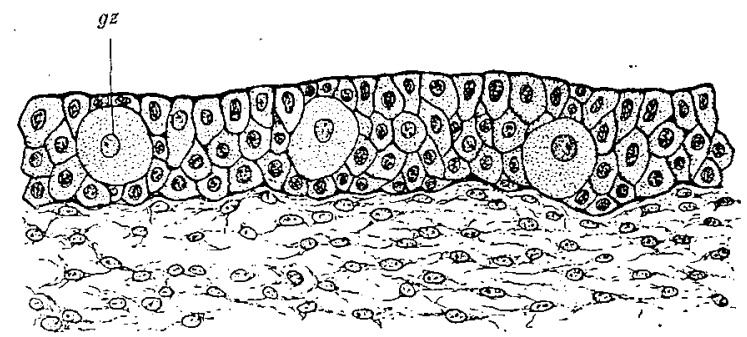

Abb. 1. Anlage der Keimdrüse in Form der Keimplatte. Schematischy).

Sexualstränge bezeichnen, wodurch der charakteristische Fortschritt gegenüber der früheren einfachen Keimplatte gekennzeichnet werden soll. Die Sexualstränge hängen weiterhin an ihrem distalen Ende mit

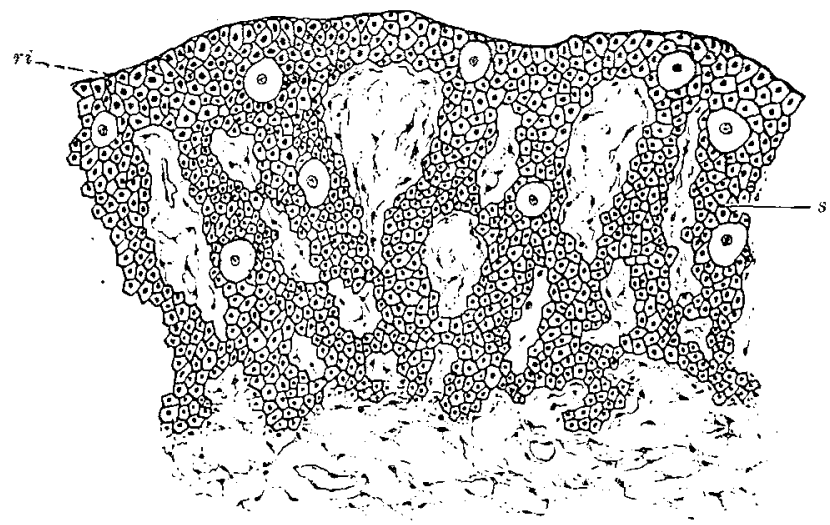

Abb. 2. Keimdrüsenanlage mit Sexualsträngen (s). Schematisch.

einem in der Tiefe zur Entwicklung gelangten epithelialen Netzwerk, dem Rete, zusammen, welches die Verbindung mit dem schon außerhalb der Keimdrüse verlaufenden Urnierengang (Wolffschen Gang) vermittelt, wodurch die Urogenitalverbindung hergestellt wird. Dieser Bau kommt bei beiden Geschlechtern in voller Utbereinstimmung zur

1) Genauere Angaben über die Textfiguren findet man in der Erklärung der Abbildungen am Schlusse der Arbeit. 
Ausbildung, so daß wir an beiden Keimdrüsen eine oberflächliche kortikale Keimplatte, tiefer gelegene medulläre Sexualstränge, Rete und Urogenitalverbindung unterscheiden können (ș. Textabb. 3 und 4). Beide Keimdrüsen zeigen den gleichen Bautypus und sind in allen ihren Anteilen vollständig homolog, wenn sie auch in diesem Zeitpunkte in manchen Besonderheiten schon voneinander abweichen.

Nun aber trennen sich die Wege ihrer weiteren Entwicklung.

Sehr einfach vollzieht sich die Differenzierung der männilichen Keimdrüse (s. Textabb. 3). Die kortikale Keimplatte nimmt keinen

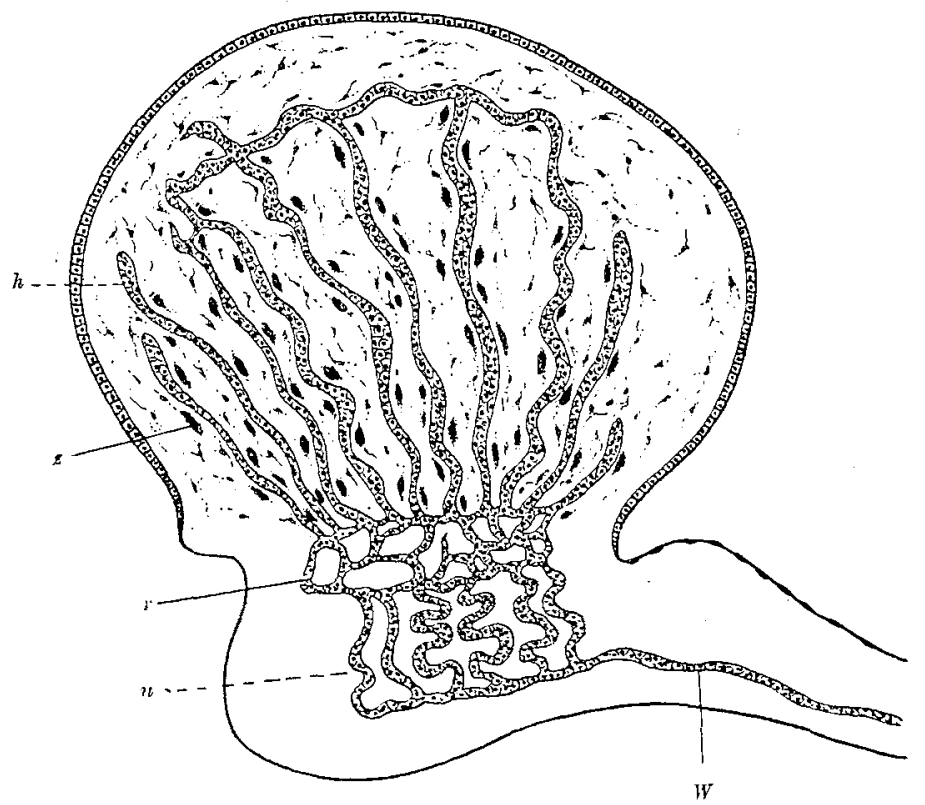

Abb. 3. Entwicklung der männlichen Keimarüse. Schematisch.

Anteil daran, sondern wird unter Schwund der eingelagerten Geschlechtszellen in einen platten Endothelbelag umgewandelt, der durch eine zusammenhängende Bindegewebsschicht (Tunica albuginea) von den Sexualsträngen abgetrennt wird. Diese allein sind es, welche die männliche Keimdrüse aufzubauen berufen sind. Sie werden zu Hodenkanälchen $(h)$, welche die Samenzellen beherbergen und durch das Rete testis $(r)$ mit den Ductuli efferentes und dem Wolffschen Gang $(W)$, dem künftigen Nebenhaden, in offene Verbindung treten. Das Organ ist in seinen Grundzügen im Markabschnitt des vorangehenden Stadiums schon ganz klar vorgezeichnet und bedàrf nur der weiteren Ausgestaltung, um als typische Hodenanlage zu erscheinen. Frühzeitig treten zwischen den Kanälchen Gruppen und Stränge großer lipoid- 
reicher Zellen auf, die Zwischenzellen (z), die in ihrer Gesamtheit bald einen ansehnlichen Bestandteil des embryonalen Hodens ausmachen.

In gerade entgegengesetzter. Weise spielt sich die Ausbildung der weiblichen Keimdrüse ab (s. Textabb. 4). Hier fällt die Hauptrolle der oberflächlichen Keimplatte zu, ja das ganze fertige Organ läßt sich im wesentlichen auf eine Wucherung derselben zurückführen, durch welche die Eistränge und weiterhin die Follikelbildungen hervorgebracht werden. Die früher so mächtige Marksubstanz aber mit ihren Sexualsträngen $(m)$ samt Rete $(r)$ und Urogenitalverbindung $(u)$ ist zur

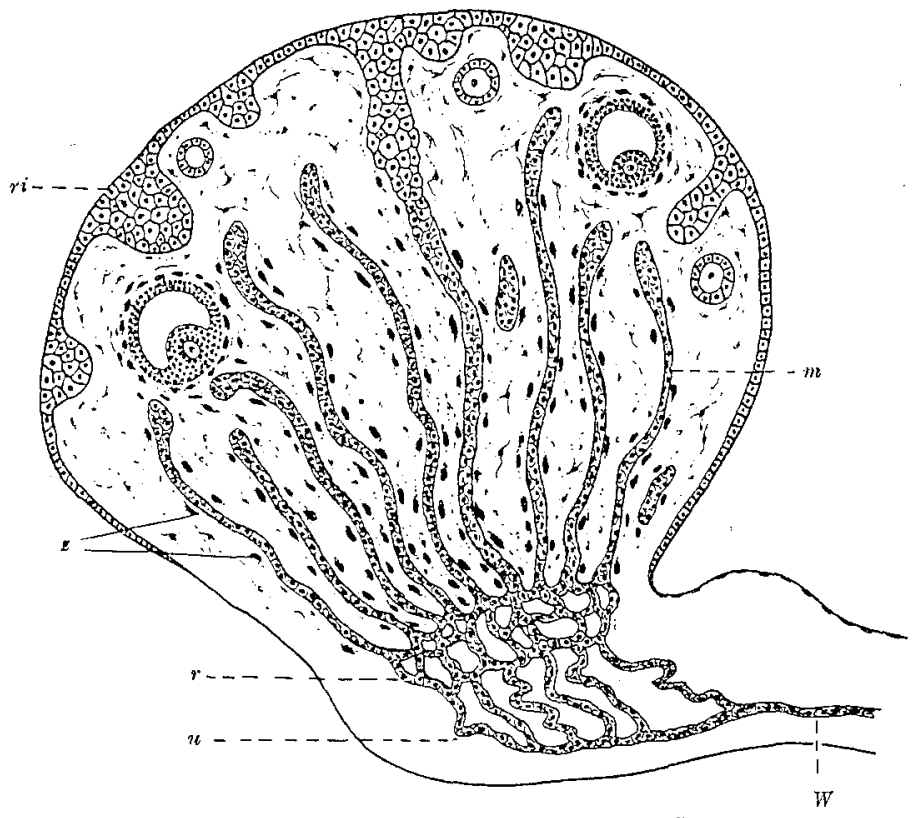

Abb, 4. Entwicklung der weiblichen Keimdrüse. Schematisch.

Bedeutungslosigkeit verurteilt und verfällt fortschreitender Rückbildung. Als dauerhaft erweisen sich nur Reste der Urogenitalverbindung, die als Epoophoron regelmäßig fortbestehen; wechseInd ist das Schicksal des Rete sowie der Sexualstränge, deren Uberbleibsel als Markstränge $(m)$ des Ovars bekannt sind und gleich den homologen Hodenkanälchen von $Z$ wischenzellen $(z)$ begleitet werden.

Diese übersichtliche Darstellung soll nur die Hauptlinien der Entwicklung vor Augen führen, um einen Vergleich zwischen männlicher und weiblicher Keimdrüse zu ermöglichen.

Bei beiden Geschlechtern erscheint zuerst eine flächenhafte Keimplatte, die aus indifferenten Epithelzellen und eingelagerten Geschlechtszellen besteht. Sie könnte als einfachster Ausdruck, als 
das Urbild einer weiblichen Keimdrüse angesehen werden. Dann treten in der Tiefe Sexualstränge samt $Z$ wischenzellen und die Urogenitalverbindung auf.

Nun folgt das Stadium der Ungleichartigkeit.

In der männlichen Keimdrüse wird die oberflächliche Keimplatte zu einem ,indifferenten Endothel rückgebildet; die Geschlechtszellen kommen ausschlieBlich in den Sexualstrüngen (Samenkanälchen) zur Reifung und werden durch das Rete und die Urogenitalverbindung in den Nebenhoden abgeführt. Nur der tiefere medulläre, distalwärts zum Wolffschen Gang gerichtete Anteil der Anlage wird für die Bildung des bleibenden Organs verwertet.

In der weiblichen Keimdrüse ist es umgekehrt. Aus der oberflächlichen Keimplatte allein gehen ihre wesentlichen Bildungen, die Eifollikel, hervor. Alle tiefer gelegenen Anteile, Sexual- (Mark-) Stränge, Rete, Urogenitalverbindung, sind für die Hauptaufgabe des Organs ohne Bedeutung und bilden sich bis auf einige Reste zurück.

Die männliche Keimdrüse ist distalwärts orientiert, die reifen samenzellen werden in den Nebenhoden abgeschoben; die weibliche Keimdrüse ist gegensätzlich gerichtet, die reifen Eizellen werden an die Oberfläche abgegeben.

Die wesentlichen Anteile der beiden Keimdrüsen sind, streng genommen, einander nicht homolog. Homolog sind vielmehr die hochwichtigen Samenkanälchen des Hodens und die funktionslosen Markstränge des Ovars; dem Rindengebiet, dem im Ovar die Hauptrolle zufällt, entspricht der indifferente Endothelbelag des Hodens.

\section{III.}

Da drängt sich wohl die Frage auf, wie dieser sonclerbare und umständliche Entwicklungsgang, insbesondere der weiblichen Keimdrüse, zu erklären sei. Die männliche Keimdrüse entwickelt sich im großen und ganzen durch eine fast geradlinige Fortbildung der embryonalen Anlage, während die weibliche im Laufe der Entwicklung einen tiefgreifenden Umbau erfährt. Was bedeutet denn die ansehnliche Mark substanz des embryonalen Ovars, die in so eigenartiger Gestaltung erscheint, um dann fast spurlos wieder zu verschwinden?

Es ist nur eine Antwort möglich. Bei unbefangener Betrachtung läßt die vollkommene Ubereinstimmung mit dem Bauplan der männlichen Keimdrüse keinen $Z$ weifel darïber aufkommen, daß die Marksubstanz des embryonalen Ovars alle Kennzeichen einer Hodenanlage trägt. Den Samenkanälchen entsprechen die Yarkstrünge, dem Rete testis das Rete ovarii, und auch die Verbindung mit dem Urnierengang (Urogenitalverbindung) findet sich hier wie dort. Die weibliche 
Keimdrüse zeigt demnach in einer frühen Entwicklungsperiode vorübergehend dieselbe Organanlage wie die männliche. Wie ist das zu erklären?

Drei Deutungsmöglichkeiten bieten sich dar. Man könnte annehmen - und es ist dies auch schon geschehen -, daß alle Keimdrüsen in ihrer Frühzeit gleichartig und gleichwertig und zwar männlich seien. Mit anderen Worten, jecler Embryo soll in einem frühesten Entwicklungsstadium zunächst männliches Gepräge zeigen; es soll anfangs nur Männchen geben, von denen erst später ein Teil zu Weibchen wird. Das ist ganz unhaltbar; denn die Geschlechtszugehörigkeit wird im Grunde nicht durch die Organform der Keimdrüsenanlage bestimmt, sondern durch die Geschlechtszellen, und diese sind, wie auch wir glauben, von allem Anfange an eindeutig bestimmt, männlich oder weiblich.

So bleibt nur die Annahme übrig, daß auch die schon weiblich determinierten Keimclrüsen vorübergehend einer Hodenanlage ähnliche Bildungen hervorbringen, die dann als rudimentäre aufzufassen wären. Das würde besagen, da $\beta$ es sich um phylogenetische Reste handelt, um Bildungen, die in ter Ahnenreibe eine Rolle spielten, deren sie im Verlaufe der Stammesentwicklung verlustig wurden. Nun tauchen ihre Spuren infolge des biogenetischen Beharrungsvermögens in der Individualentwicklung immer wieder auf, um mangels funktioneller Bildungsreize allmühlich wieder zu verkümmern.

Aber da sind wieder zwei Möglichkeiten ins Auge zu fassen.

Vielleicht war die weibliche Keimdrüse ferner Ahnen ähnlich gebaut und orientiert wie die männliche; vielleicht schob auch sie ihre reifen Keimzellen distalwärts ab. Das ist kaum anzunehmen. Es ist ganz unwahrscheinlich, daß die reifen Eier durch ein Rete in den Urnierengang gelangt sein sollten, und ganz unverständlich wäre dann das Auftreten des Müllerschen Ganges auch beim männlichen Embryo, wenn der Wolffsche Gang den Keimdrüsen beider Geschlechter als Ausführungsgang geclient hätte.

Die letzte und annehmbarste Deutung ist die, daß der Markanteil ! des embryonalen Ovars wirklich nichts anderes als eine rudimentäre Hodenanlage darstellt, so daß wir in Úbereinstimmung mit zahlreichen früheren Autoren (Waldeyer, Fgli, Janošik, Meixner, Sauerbeck u. a.) der weiblichen Keimdrüse eine bisexuelle, hermaphroditische Anlage zuerkennen müßten. Tatsächliche Bisexualität ist aber ausgeschlossen, wenn wir auf dem Standpunkte stehen, daß die Geschlechtsbestimmung mit der Befruchtung endgültig vollzogen sei. Dann kann es sich also nicht um funktionelle, sondern nur um formale Bisexualität handeln, nicht um bisexuelle Gegenwart, sondern nur um bisexuelle Vergangenheit, nicht um aktuelle, sondern nur um geschichtliche, um Ahnenzwittrigkeit, deren unvergängliche Spuren auch jetzt noch in der Ontogenese regelmäßig zum Vorschein kommen. 
In guter Ubereinstimmung mit dieser Annahme finden wir auch die beiden Ausfuhrwege der Keimdrüsen - Müllerschen und Wolff schen Gang samt der Urogenitalverbindung - bei beiden Geschlechtern angelegt, and viele andere Anzeichen einer bisexuellen Anlage zeigt nicht nur das Genitalsystem, sondern auch der Gesamtorganismus.

Man darf also Markstränge und Rete des embryonalen Ovars aus guten Gründen als ein hodenähnliches Organrudiment, als ein Testoid ansehen, aber nicht als eine echte männliche Keimdrüsenanlage, da ihr die Möglichkeit männliche Keimzellen hervorzubringen, abgesprochen werden muß. Nur der äußerliche Bautypus, nur die Organform eines Hodens wird nachgeahmt, ohne jegliche geschlechtsspezi-

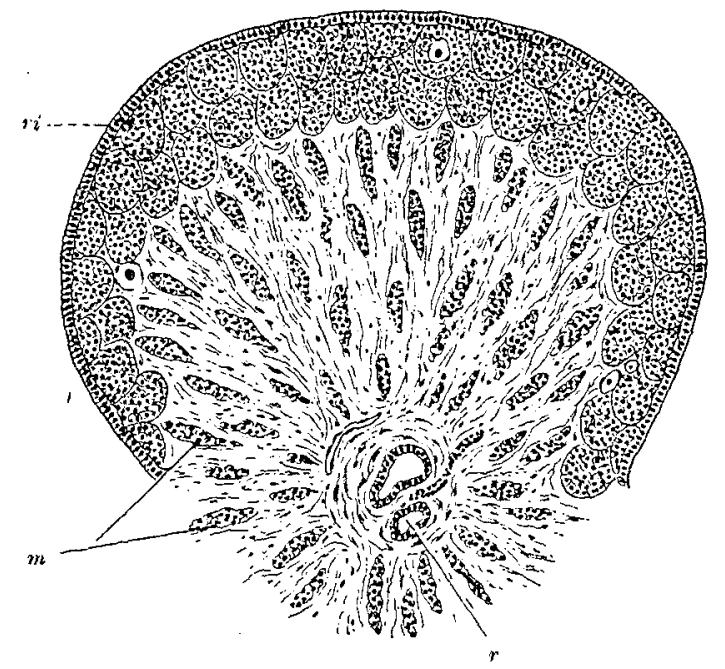

Abb. 5. Ovarium eines jungen Fuchses (etwa 4 Monate) nach Buhler. " Mrarkstränge, ri Rinde, $r$ Rete.

fische Funktionsmöglichkeit. Niemand wird von diesem testoiden Gebilde erwarten dürfen, daß es Samenzellen tragen werde; denn das Geschlecht und damit insbesondere die Geschlechtszellen sind rom Anbeginn der Ontogenese eindeutig bestimmt. Darum kann nur eine Art von Geschlechtszellen zur Entwicklung gelangen, und wenn in diesem testóiden Formgehäuse Keimzellen überhaupt auftreten, so können es demnach auch auf diesem fremdartigen Boden nur weibliche sein. Tatsächlich entwickeln sich auch in fetaler Zeit die Geschlechtszellen der Markstränge zu ansehnlichen Eizellen, wodurch absonderliche, unförmliche follikelähnliche Bildungen, die sogenannten Markfollikel, zustande kommen. Eine lange Lebensdauer ist ihnen aber nicht beschieden, und meist schwinden außer ihren Geschlechtszellen auch die Markstränge selbst schon frühzeitig und spurlos. 
Bei manchen Säugetieren aber bleiben neben Rete und Urogenitalverbindung auch Reste der Markstränge in ziemlicher Ausdehnung lange (s. Textabb. 5) oder selbst dauernd erhalten.

In ungewöhnlicher Deutlichkeit bewahrt z. B. das Ovar des Maulwurfs zeitlebens den embryonalen Typus. Schon äußerlich läßt es eine gewisse $Z$ weiteilung erkennen, die bereits Treviranus erwähnt, und an Durchschnitten zeigen die beiden Abschnitte einen grundverschiedenen Bau, den Leydig in seinem klassischen Lehrbuche der Histologie im Jahre 1857 schon recht anschaulich beschreibt (s. Abb. 1 und 3 auf Taf. VI).

Nur eine oberflächliche kappenförmige Rindenzone wird von echtem Ovarialgewebe eingenommen; die meist größere Markschicht aber besteht aus wenig differenzierten, kurzen, zylindrischen Epithelsträngen und reichlichen Zwischenzellen (s. Abb. 5 auf Taf. VI). Am Rande erscheinen Retekanälchen (s. Abb. 4 auf Taf. VI), die mit dem Epoophoron zusammenhängen. Warum Tourneux ${ }^{1}$ ) angesichts eines solchen Befundes von physiologischem Hermaphroditismus sprach, liegt auf der Hand. Die Entwicklung, Lage, Bauart und Anordnung, sowie die Anwesenheit zahlreicher $Z$ wischenzellen läßt keinen Zweifel darüber aufkommen, daß hier ein mächtiges Testoid vorliegt (s. Abb. 1 und 3 auf Taf. VI), durch welches eine Bisexualität der Keimdrüse leicht vorgetäuscht werden kann. Gleichwohl handelt es sich nur um eine rein weibliche Gonade, in welcher regelmäßig die fetalen Sexualstränge dauernd erhalten bleiben. Die hermaphroditische Ahnenform der Keimdrüse bleibt bestehen, ohne den unisexuellen Geschlechtscharakter irgendwie zu beeinträchtigen; denn die Persistenz der heterosexuellen Rudimente ward hier zur Norm²).

Anders liegen die Verhältnisse dann, wenn solche bisexuelle Atavismen auch dort gefunden werden, wo sie in der Regel zu verschwinden bestimmt sind (s. Abb. 2 auf Taf. VI).

\section{IV.}

Wir wissen, daß im allgemeinen bei Mensch und Säugetier die heterosexuellen Rudimente der Rückbildung anheimfallen. Von den Vorgängen, die sich dabei abspielen, kann man sich etwa folgende Vorstellung machen. Jede Keimdrüse wird auf Grund des von den Ahnen ver-

1) Bibliographie anatomique. Supplém. 1904.

2) Mit Unrecht bestreiten Tandler und Grosz (Die biologischen Grundlagen der sekundären Geschlechtscharaktere, 1913) die Richtigkeit der Beschreibung von Tourneux. Ihre Behauptung, daß im Testoid nur "Anhäufungen von Zwischenzellen \& zu finden seien, ähnlich "wie am Ovar des Pferdes oder des Esels \& ist unzutreffend. 
erbten Bauplanes in $Z$ witterfor $m$ angelegt. Der geschlechtsspezifische unisexuelle Differenzierungsimpuls aber wird diesem durch phylogenetische Gestaltungsfaktoren hervorgerufenen, zwittrigen Organbau durch die individuell erworbenen geschlechtsbestimmenden Faktoren aufgezwungen. Die Geschlechtszellen sind von allem Anfange an weder bisexuell noch indifferent, sondern unisexuell und verschaffen dadurch im weiteren Fortgange der Entwicklung der ihrem Geschlechtsvorzeichen entsprechenden Komponente in dem zwitterig angelegten Keimdrüsenbau das Übergewicht, so daß sich dieser nun vorwiegend in ausgesprochen männlicher oder weiblicher Richtung differenziert.

Oder mit anderen Worten: Bei der Differenzierung der Keimdrüse sind zwei einander teilweise widerstrebende Richtungen im Spiele; die phylogenetische automatische Bisexualität wird von der lebendig wirksamen ontogenetischen Unisexualität bekämpft. Die phylogenetische, von den Urahnen vererbte Entwicklungstendenz sucht mit wunderbarer Beharrlichkeit die » zwittrige Stammform « (Sauerbeck $\left.{ }^{1}\right)$ ) zu erhalten; die ontogenetische Tendenz aber ist darauf gerichtet, den eindeutigen Geschlechtscharakter zur Vorherrschaft zu bringen. Sie drängt die heterologe Komponente der formalen Zwitteranlage zurück und läßt nur das zur vollen Reife kommen, was ihrem Geschlechtsvorzeichen entspricht und zu funktioneller Wirksamkeit berufen erscheint.

Wie das geschieht, welche Faktoren dabei wirksam sind, das entzieht sich unserer Einsicht. Frühzeitig scheint sich der unisexuelle Einfluß in der Gestaltung der Keimdrüse selbst durchzusetzen, und allmählich - vielleicht schon unter ihrer endokrinen Mitwirkung - erhält der Gesamtorganismus seinen eindeutigen Geschlechtscharakter in somatischer und psychischer Hinsicht. Es ist selbstverständlich, daß die Ontogenese Siegerin bleibt; sonst wäre sie ja nicht » Ontogenese ", nicht der typische Ablauf der individuellen Entwicklung. Aber restlos vermag sie die heterosexuelle Komponente der atavistischen $Z$ witteranlage doch nicht zu verdrängen; Spuren bleiben, wenn sie auch im allgemeinen geringfügig sind und meist in Verborgenheit verharren.

Wenn aber die ontogenetische unisexuelle Gestaltungskraft aus irgendwelchen Ursachen - etwa Insuffizienz der geschlechtsbestimmenden Faktoren u. a.m. - unzureichend ist, dann kommt der eindeutige Geschlechtscharakter nicht mit voller Klarheit und Bestimmtheit zum Ausdruck, und die heterosexuelle Komponente der atavistischen Zwitteranlage drängt sich, ungenügend gehemmt ${ }^{2}$ ), hervor. Es kommt dann zu jenen abnormen Erscheinungen, die man als hermaphroditische bezeichnet. Heterologe Bildungen treten auf, die man von unserem Stand-

1) Frankf. Zeitschr. f. Pathol. 3. Bd. 1909.

2) Vgl. Herbst, C., Formative Reize. Leipzig 1901. 
punkte aus nicht für pathologische Neuerwerbungen halten darf, sondern für atavistische Rückbleibsel, die sich erhalten und infolge ungenügender Hemmung weiterentwickelt haben. Wenn beim Manne Gebilde gefunden werden, die als Eileiter oder Utterus gedeutet werden müssen, wird man mit Recht von Persistenz der Müllerschen Gänge sprechen. Wenn in einer weiblichen Keimdrüse hodenähnliche Bildungen angetroffen werden, wird man folgerichtig auch nicht an Neubildung, sondern an abnorme Beständigkeit und Weiterbildung der Sexual(Mark-) Stränge denken müssen. So wird man das Bild der Ovotestes, der Zwitterdrüsen, aufzufassen haben, in denen, ganz ühnlich wie im Ovarium des Maulwurfs, ein zum Rete hin orientiertes Netzwerk von Zellsträngen, das ganz und gar einem unfertigen Hoden ähnelt, von einer unverkennbaren Ovarialzone skappenartig« (Sa uerbeck, L. Pick) überdacht wird (s. Abb. 2 auf Taf. VI). In diesem Falle spricht man aber gewöhlich von Hermaphroditismus gland ularis oder verus, weil hier nach sehr verbreiteter Anschaung eine wahreZwitterdrüse vorliegen soll. Um Scheinzwittertum (Pseudohermaphroditismus) aber soll es sich dann handeln, wenn die Keimdrüse selbst eindeutig ist und das Zwittertum sich nur am übrigen Genitalsystem (Leitungswege, äußeres Genitale) oder in sonstiger somatischer oder psychischer Heterologie kundgibt. Wahres und falsches Zwittertum werden meist scharf unterschieden; denn nur beim Hermaphroditismus verus findet sich eine zwittrige Keimdrüse, von der angenommen wird, daB sie möglicherweise beide Arten von Gameten hervorbringen könnte.

Diese scharfe Unterscheidung ist unbegründet. In dem einen wie im anderen Falle handelt es sich um wesentlich gleichartige Mißbildungen; die Drüsenzwittrigkeit ist nicht echter als das sonstige Scheinzwittertum. Ein wahrer Hermaphroditismus würde - hierin stimme ich Kermauner ${ }^{1}$ ) zu - die Erzeugung geschlechtsverschiedener Gameten oder doch die grundsätzliche Möglichkeit zweifacher Keimzellbildung zur Voraussetzung haben. Das ist hier nicht zu erwarten; denx die beiden Anteile der Zwitterdrüse sind nicht so gleichwertig, wie man anzunehmen pflegt, und nicht in gleichem Maße befähigt, ihrer Bauart entsprechende Keimzellen hervorzubringen. Wenn nämlich das Geschlecht schon im Beginne der Ontogenese bestimmt ist, so kann nur der eine Anteil dem vorgezeichneten, eingeschlechtigen Gestaltungsplan entsprechen, der andere aber verdankt sein Dasein im Grunde nur der automatischen, ungehemmten Wiederkehr einer altvererbten Organform und nicht, wie man vielfach glaubt, einer abnormen Neu- oder Mehrbildung andersgeschlechtigen Charakters.

Die Ontogenese ist geschlechtsbestimmt und sucht die Keimdrüse

1) Frankfurt. Zeitschr. Bd. 11. 1912. 
ihrem Vorzeichen gemäß zu gestalten. Das gelingt ihr aber in diesen pathologischen Fällen nur unvollkommen, und andererseits wird auch die heterologe Komponente der bisexuellen Anlage nicht genügend niedergehalten. So kommt das zwittrige Aussehen solcher Keimdrïsen zustande. Aber das Zwittertum ist nur Schein, und es wird auch hier, wenn überhaupt, nur eine Art von Keimzellen geben, wo immer auch sie in der $Z$ witterdrüse angetroffen werden. Mögen sie im ovariellen oder testoiden Anteile gefunden werden, ihr Vorzeichen wird immer das gleiche sein, genau so wie Rindenteil und Markstränge des embryonalen Ovars nur Geschlechtszellen der gleichen Art beherbergen. Das Geschlecht ist längst bestimmt, und das atavistische Gehäuse vermag aus sich heraus ebensowenig andersgeschlechtige Keimzellen hervorzubringen oder das gegebene Vorzeichen abzuändern, wie die Markstränge des embryonalen Eierstockes oder das Testoid des Maulwurfovars Samenzellen zu liefern imstande sind.

Das Geschlecht ist also wohl auch bei solchen Scheinzwittern zumeist eindeutig bestimmt, aber offenbar in unzureichendem Grade. Woran das liegt, können wir nicht angeben; konstitutionelle Faktoren wie konditionelle Umstände könnten dabei im Spiele sein. Bezeichnend ist der Umstand, daß nicht nur die heterosexuellen Bildungen sich hervorzudrängen vermögen, sondern auch die autosexuellen ihre volle formale und funktionelle Ausgestaltung nur selten erreichen. In den Hoden von Pseudohermaphroditen kommt es gewöhnlich nicht zur Spermatogenese, der Hoden von Kryptorchen bleibt meist infantil, der Ovarialteil der Ovotestes trägt nur ausnahmsweise reife Follikel. Das alles spricht für eine mangelhafte, unvollkommene und unscharfe Geschlechtsprägnanz, für eine schwächliche, unzureichende Unisexualität, welche den eigenen Geschlechtscharakter nicht zur vollen Höhe und das Gegensätzliche nicht zu entsprechender Rückbildung zu bringen vermag.

So kann sich das Nebeneinander von Wolffschem und Müllerschem Gang ergeben, und so kann es auch zur Entstehung von scheinbar zwittrigen Keimdrüsen (Ovotestes) kommen. Man muß sich aber immer vor Augen halten, daß die zwittrige Organform der Keimdrüse an sich (man denke an das Ovarium des Maulwurfs) nicht ausschlaggebend sein kann für die Zuerkennung wahrer Zwittrigkeit; denn der bisexuelle Organtypus gelangt rein automatisch auf Grund des ererbten Entwicklungsplanes zur Anlage, während die Geschlechtszugehörigkeit einzig und allein vom Charakter der Geschlechtszellen bestimmt wird.

Hermaphroditismus verus wie spurius sind demnach im wesentlichen gleichartige Mißbildungen frühesten Ursprunges und gehören in eine und dieselbe Kategorie, wie mit Sauerbeck auch L. Pick ${ }^{1}$ ) an-

1) Arch. f. mikr. Anat. 84. Bd. 1914. (Mit reichhaltigem Literaturnachweise.) 
nimmt, sind Glieder derselben Reihe, die vom mangelhaften Descensus und Kryptorchismus, von unterentwickelten Keimdrüsen mit allen ihren somatischen und psychischen Begleiterscheinungen bis zur scheinzwittrigen Keimdrüse führt. Bei jeder der erwähnten Formen unvollkommener und unscharfer Unisexualität kann infolge der bisexuellen Grundlage (der 》zwittrigen Stammform « - Sauerbeck $\left.{ }^{1}\right)$ ) des Organismus die somatische und psychische Eindeutigkeit, die wir unter normalen Verhältnissen als typischen Geschlechtscharakter kennen, in verschiedenem Grade abgeschwächt und verwischt erscheinen, ohne daß wir derzeit noch eine bestimmte Gesetzmäßigkeit zu erkennen vermögen. Bei Individuen mit völlig eindeutigen, aber unterentwickelten, minderwertigen Keimdrüsen kann unter Umständen die Zwittrigkeit des Organismus in einem solchen Grade ausgesprochen sein, daß die Geschlechtszugehörigkeit fast ebenso unbestimmbar werden kann wie bei Fällen mit sogenannten Zwitterdrüsen. Infolge der Insuffizienz der unisexuellen Determinanten kommt das atavistische Doppelwesen mehr minder kräftig zum Durchbruch, und das Endergebnis ist, wenn auch nicht eine Zweigeschlechtigkeit im funktionellen Sinne, so doch - infolge der Persistenz und Fortentwicklung heterosexueller Anlagen ein Wirrwarr, ein Durcheinander der Geschlechtsmerkmale, eine Vermischung gegensätzlicher Eigenschaften, eine weitgehende Verschwommenheit und "Verwischung" (Orth) des eigentlichen Geschlechtscharakters, wodurch die mannigfachen Typen der hermaphroditischen Reihe bei aller Verschiedenheit doch einen gewissen gemeinsamen Grundzug erhalten.

Alle diese Erscheinungen, so wechselvoll sie sich auch darbieten, scheinen auf das gleiche ursächliche Moment der mangelhaften Geschlechtsbestimmtheit zurückzugehen. Sie stellen früheste Entwicklungsstörungen dar, Mißbildungen frühesten Ursprunges, als deren gemeinsames Kennzeichen gelten kann: mangelhafte unisexuelle Prägung in formaler und funktioneller Hinsicht, mit der in der Regel ein verschiedengradiges Hervortreten heterosexueller Merkmale als Folge der atavistischen bisexuellen Anlagge verknüpft ist.

Diese Auffassung scheint den Tatsachen besser zu entsprechen als jene, welche in den Ovotestes den Ausdruck eines.echten Hermaphroditismus, einer potentiellen Bisexualität, sieht. Die Anhänger eines solchen Hermaphroditismus verus nehmen an, daß die Ovotestes durch die Vereinigung zweier heterogener Anteile, die aus gesonderten Bezirken des Keimepithels hervorgehen ${ }^{2}$ ), zustande kommen und somit tatsächlich aus männlichem und weiblichem Keimdrüsengewebe aufgebaut

1) Frankfurter Zeitschr. f. Pathologie. 3. Bd. 1909.

2) Kopsch und Szymonowiez, Anat. Anz. 12. Bd. 1896. 
werden. Es sollte sich demnach um wahre Zwitterdrüsen handeln, in denen sowohl männliche wie weibliche Gameten oder doch deren Vorstufen gebildet werden könnten ${ }^{1}$ ). Die Träger solcher Keimdrüsen müßten dann mit vollem Rechte als echte Zwitter bezeichnet werden.

Was gegen diese Auffassung spricht, ist im Voranstehenden wiederholt angeführt worden. Ich betone nur nochmals den von allen Beobachtern hervorgehobenen charakteristischen Bau der Ovotestes, der mit seiner gesetzmäßigen Lagerung der beiden Anteile so eindringlich an das embryonale Ovar und an das Maulwurfovarium erinnert und so überzeugend für die Ableitung aus der bisexuellen Anlage der embryonalen Keimdrüse spricht (s. Textabb. 2-5 und Abb. 1-3 auf Taf. VI).

Wir halten demnach die $Z$ witterdrüsen der Säugetiere nicht, wie L. Pick u. a., für zweigeschlechtige, sondern für unvollkommene unisexuelle Keimdrüsen, in denen die heterologen Rudimente der bisexuellen Anlage nicht nur keine genügende Hemmung und Rückbildung, sondern sogar eine abnorme Weiterbildung erfahren haben.

Damit wird ein gewisser einheitlicher Gesichtspunkt für die Beurteilung des Hermaphroditismus festgehalten, der allerdings im einzelnen, anatomisch wie ätiologisch, noch weiterer Klärung bedarf, zumal manche Typen, wie z. B. die Halbseitenzwitter, im Rahmen dieser Darstellung zunächst unberücksichtigt geblieben sind (s. S. 112).

Die Ursachen hermaphroditischer Mißbildung kennen wir nicht. Ob sie in der Beschaffenheit der elterlichen Keimzellen zu suchen sind oder in anderen mitbestimmenden Faktoren und Umständen, ist uns derzeit gänzlich unbekannt.

V.

Auf eigenartige Weise sucht Steinach das Wesen des Hermaphroditismus zu erklären. Nach ihm ist die Ursache im gemeinsamen Vorhandensein geschlechtsverschiedener $Z$ wischenzellen zu suchen: das Männchen hat männliche, das Weibchen weibliche Zwischenzellen, und in der Keimdrüse des Hermaphroditen sollen beide Arten von Zwischenzellen neben einander vorkommen. Dabei kann der generative Anteil selbst ganz eindentig erscheinen, wodurch der Pseudohermaphroditismus leicht verständlich würde. Manche Autoren sind auch schon bereit, neben einem generativen Hermaphroditismus der Keimdrüsen einen interstitiellen anzuerkennen, somit eine neue Art von Zwitterdrüse, in der nicht verschiedengeschlechtige generative Anteile, sondern nur geschlechtsverschiedene $Z$ wischenzellen sich vereint finden sollen. Allerdings hat noch niemand dergleichen mit Bestimmtheit gesehen. Für Steinach und seine Anhänger gibt es aber im Grunde nur eine

1) L. Pick, Archiv f. mikr. Anat. 84. Bd. 1914, u. a. 
Art von Hermaphroditismus, den interstitiellen; denn nach ihrer Meinung sind die Zwischenzellen allein für den Geschlechtscharakter verantwortlich ${ }^{1}$ ). Nur die Zwischenzellen sollen den Geschlechtscharakter des Soma wie den der Psyche bestimmen; nur durch sie werde die ursprünglich asexuelle Embryonalanlage veranlaßt, sich in männlicher oder weiblicher oder gemischter Richtung zu differenzieren.

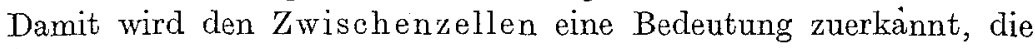
weit über unsere frühere Bewertung hinausgeht. Was sind diese vielgenannten Zwischenzellen?

Wir haben sie bereits öfters erwähnt. Schon in der embryonalen Keimdrüse treten sie zwischen den Sexualsträngen auf und werden zum bleibenden Bestandteil des Hodens, wo sie in Ballen und Zügen zwischen den Samenkanälchen verteilt sind. Wenn in der weiblichen Keimdrüse Markstränge bestehen bleiben, werden auch diese häufig von Zwischenzellen begleitet, was man besonders schön im Maulwurfovar sehen kann (s. Abb. 5 auf Taf. VI). Sonst finden sie sich in den Ovarien geschlechtsreifer Säugetiere in verschiedenem Ausmaße, und regelmäßig umlagern sie die größeren Follikel als sog. Thekazellen.

Die Zwischenzellen sind Zellen eigener Art, die nur den Keimdrüsen eigentümlich sind. Sie sind ziemlich groß, treten selten vereinzelt, meist in Gruppen und Strängen auf und enthalten reichliche Einlagerungen, vor allem Lipoidsubstanzen, wodurch die Zellen häufig ein wabiges Aussehen erhalten und den Rindenzellen der Nebenniere ähnlich werden, ferner Pigment und im menschlichen Hoden auch Kristallbildungen (Reinke).

Diese Zellen sind lange bekannt, von Leydig im Jahre 1850 entdeckt und nach ihm auch Leydigsche Zellen genannt worden; aber erst in neuerer Zeit, seit die innersekretorischen Vorgänge im Organismus mehr Beachtung fanden. hat man ihnen größere Aufmerksamkeit geschenkt.

Wir wissen, daß dit Keimdrüsen nicht nur der Fortpflanzung dienen, sondern indirekt durch eine Art Fernwirkung auch den Gesamtorganismus, Körper und Seele, geschlechtsspezifisch zu stimmen vermögen: Daß der Mann das charakteristische männliche und das Weib weibliches Wesen zeigt, daran sind zweifellos die Keimdrüsen in hervorragender Weise mitbeteiligt; denn der Verlust oder gewisse Erkrankungen derselben rufen auffallende Abweichungen vom normalen Geschlechtstypus hervor.

Es sollen nun gerade die in tergenerativen Elemente, die Zwischenzellen, mit dieser zweiten Aufgabe der Keimdrïse betraut sein, durch

1) Steinach, E., Pubertätsdrüsen und Zwitterbildung. Arch. f. Entw.-Mech. 42. Bd. 1916: »Es gibt für alle Zwittererscheinungen nur eine Ursache und diese 'beruht auf dem Entstehen einer zwittrigen Pubertätsdrüse ... 
innere Sekretion den allgemeinen Geschlechtscharakter, die sog. sekundären Sexualmerkmale, zur Entfaltung zu bringen und zu erhalten.

Besonders sind es französische Autoren, vor allem Bouin und Ancel1), gewesen, die diese Lehre durch Beobachtung und Versuch zu befestigen suchten. Ihnen schlossen sich vicle andere an, so auch Tandler und Grosz ${ }^{2}$ ), Bied ${ }^{3}$ ) und mit sehr interessanten Versuchen in neuerer Zeit Steinach4).

Nur einige der Tatsachen, die dieser Auffassung zugrunde liegen, will ich kurz anführen.

Es hat sich herausgestellt, daß die volle Ausbildung der sekundären Geschlechtsmerkmale nicht so strenge, wie man glauben könnte, an die vollkommene Ausbildung fortpflanzungsfühiger Keimdrüsen gebunden ist. Die Beobachtungen betreffen meist das männliche Geschlecht, bei dem der Geschlechtscharaliter viel bestimmter und positiver zum Ausdruck gelangt als beim weiblichen, und ergaben, daß, im Gegensatze zu Kastraten, häufig auch solche Männchen ein entschiedenes männliches Wesen an den Tag legen, deren Hoden nur minderwertige, zur Samenbildung unfähige generative Anteile enthalten, wobei aber die Zwischenzellen meist in ungewöhnlicher Venge vorhanden sind. Röntgenisierte, pathologisch veränderte und verpflanzte Keimdrüsen sowie namentlich Hoden von kryptorchen Menschen und Säugetieren lieferten das Beweismaterial.

Einen Schritt weiter ging Steinach5). Er kastrierte junge, geschlechtsunreife Tiere, zumeist Meerschweinchen, und pflanzte ihnen heterologe Keimdrüsen ein, also den frühkastrierten Münnchen Ovarien, den Weibchen Hoden. Wenn diese gut einheiIten, vollzog sich eine allmähliche Umstimmung des Geschlechtscharakters, eine künstliche Feminierung und Maskulierung. Die operierten Weibchen trugen schließlich in Körperbau und Gebaren ein ausgesprochen männliches Wesen zur Schau, die Männchen wurden feminin und konnten sogar, da ihre Mamma drüsenreich und sekretionsfähig wurde, junge Meerschweinchen säugen, was sie überdies mit Geschick und Befriedigung taten.

Diese Versuche erregten mit Recht großes Interesse. Die Deutung, die Steinach ihnen gab, hob das Ansehen der $Z$ wischenzellen noch weit über das bisherige Maß. Da in den verpflanzten Keimdrüsen die generativen Anteile (Follikel, Samenkanälchen) meist stark unterentwickelt und rückgebildet erschienen, die $Z$ wischenzellen dagegen

1) Arch. Zool. exp. et gén. I. 1903.

2) Die biologischen Grundlagen der sekundären Geschlechtscharaktere. Berlin, Springer, 1913.

3) Innero Sekretion. 3. Aufl. 1916.

4) Zentralbl. f. Physiol. 24. Bd. 1910.

5) Zentralbl, f. Physiol. 25. Bd. 1911. 
sehr gewuchert waren, schrieb Steinach diesen die geschlechtsumstimmenden Wirkungen zu. So sollen also die Zwischenzellen nicht nur den sekundären Geschlechtscharakter ihres normalen Trägers bedingen, sondern bei gelungener Übertragung auch einem andersgeschlechtigen (kastrierten) Individuum ihren Geschlechtscharakter aufzuzwingen imstande sein, wobei selbst eine über die Norm hinausgehende Steigerung erzielt werden kann, was durch die Milchsekretion ohne vorangehende Gravidität bewiesen zu sein scheint. » Pubertätsdrüse «nannte S tein ach die Gesamtheit der Zwischenzellen, da auch die Geschlechtsreifung von ihnen herbeigeführt werden soll.

Auch männliche und weibliche Keimdrüsen wurden einem frühkastrierten männlichen Meerschweinchen gleichzeitig eingepflanzt und so ein hermaphroditisches Tier erzeugt, dessen geschlechtliche Neigungen sich bald in männlicher, bald in weiblicher Richtung betätigten. Hier waren neben reduzierten generativen Anteilen beide Arten von Zwischenzellen reichlich im Implantat nachweisbar, wodurch Steinach zu der Auffassung bestimmt wurde, daß der Hermaphroditismus überhaupt durch Mischung geschlechtsverschiedener Zwischenzellen verursacht werde.

Aber die Leistungen der $Z$ wischenzellen sollen sich nicht darauf beschränken, den sekundären Geschlechtscharakter hervorzubringen, die Geschlechtsreife auszulösen und geschlechtliche Umstimmung bewirken zu können, Steinach und seine Anhänger versteigen sich schließlich zu der verblüffenden Behauptung, daß die Anlage des Embryo zunächst weder bisexuell noch unisexuell, sondern geschlechtlich unbestimmt, asexuell sei und erst durch die Zwischenzellen veranlaßt werde, sich in männlicher oder weiblicher Richtung zu differenzieren ${ }^{1}$ ).

Damit sind unsere herkömmlichen Anschauungen so gründlich auf den Kopf gestellt, daß eine umfassende kritische Prüfung der Sachlage angezeigt wäre. Ich will mich aber hauptsächlich nur mit jenen Punkten befassen, die mit meinen Ausführungen in Zusammenhang stehen und im übrigen bloß meiner Utberzeugung dahin Ausdruck geben, daß für die neue 》 Pubertätslehre « vollgültige Beweise bisher nicht erbracht worden sind.

Bei allen Beobachtungen und Versuchen, welche die ausschließliche Bedeutung der Zwischenzellen für die Entfaltung und Erhaltung des Geschlechtscharakters erhärten sollen, fanden sich außer ihnen auch noch generative Anteile, wenn auch in unreifem oder verkümmertem Zustande. Es fehlt der zwingende Beweis dafür, daß die endokrinen Wirkungen nicht von diesen ihren Ausgang nehmen könnten ${ }^{2}$ ). Selbst

1) Zentraliol. f. Physiol, 27. Bd. 1913.

2) Vgl. auch Bucura (Zentralbl. f. Gynäk. 1913) und insbesondere die inzwischen erschienene Arbeit Stieves über die Zwischenzellen im Hoden der Dohle (Arch. f. Entw. 45. Bd. 1919). 
in jenen Fällen, wo in den verpflanzten Keimdrüsen die generativen Teile eine weitgehende Reduktion und Einschmelzung erfahren, kann weder den verbliebenen Resten, noch dem resorbierten generativen Material jede Wirkungsmöglichkeit abgesprochen werden. Kurz, der Nachweis, daß wirklich nur die $Z$ wischenzellen die beobachteten Wirkungen hervorrufen, ist durch die Versuche Steinachs nicht erbracht worden, da er nicht reine, »isolierte Pubertätsdrüsen《, sondern Keimdrüsen, die auch reichlich generative, epitheliale Bildungen enthielten, zur Einheilung brachte ${ }^{1}$ ).

Der unglückliche Name "Pubertätszellen" wurde den Zwischenzellen von Steinach beigelegt, weil er sie auch für die " Entwicklung der somatischen wie der psychischen Pubertät « verantwortlich macht. Dazu ist folgendes $\mathrm{zu}$ bemerken.

$\mathrm{Zu}$ einer für jede Spezies im allgemeinen fest bestimmten Frist tritt die Geschlechtsreife ein (ebenso wie die Geburtsreife, das Klimakterium und der Tod), aber nichts beweist, daß dabei die Zwischenzellen eine ausschlaggebende Rolle spielen. Kryptorche wie Pseudohermaphroditen besitzen in ihren Hoden nicht selten recht ansehnliche s Pubertätsdrüsen ", ohne deshalb immer auch die charakteristischen Merkmale der Geschlechtsreife zu erlangen.

Noch weniger wird man sich mit der Vorstellung befreunden können, daß die Anlage des Embryo weder unisexuell noch bisexuell, sondern asexuell sei (Lipschütz) und daß die geschlechtliche Differenzierung erst nachträglich durch Vermittlung der Zwischenzellen erfolgen solle. Wir halten daran fest, daß das Geschlecht des Embryo schon im einzelligen Ausgangsstadium festgelegt sei und es somit eine asexuelle Anlage im allgemeinen gar nicht geben könne. Die gleichzeitig mit der Befruchtung geweckte ontogenetische, unisexuelle lebendige Gestaltungskraft muß sich jedesmal, in jedem Einzelfalle gegen eine stammvererbte, gleichsam automatisch wirkende bisexuelle Entwicklungsrichtung durchsetzen, so daß man mit einem gewissen Rechte von einer unisexuellen Individual- und einer bisexuellen Stammesanlage, aber keineswegs von, einem asexuellen Embryonalstadium sprechen könnte.

Gerade die früher erwähnten Halbseitenzwitter, die in der hermaphroditischen Reihe eine Sonderstellung einnehmen, möchte ich als Beleg für diese Annahme verwerten. Wenn in der ursprünglich asexuellen Anlage eines Embryo die spätere geschlechtsspezifische Differenzierung des Soma ausschließlich durch die endokrine, den Gesamtorganismus beeinflussende Wirkung der Zwischenzellen hervorgerufen werden sollte, so wäre die seltsame Erscheinung des Halbseitenzwitters

1) Man vergleiche die Abbildungen auf Tafel XIX des Aufsatzes: Pubertätsdrüsen und Zwitterbildung. Arch. f. Entw.-Mech. 42. Bd. 1916. 
mit der scharfen Abgrenzung einer männlichen und weiblichen Körperhälfte kaum verständlich. Die beiden heterogenen geschlechtsdifferenzierenden Hormone müßten hier doch erst recht eine Verwischung der Geschlechtsmerkmale herbeiführen, wie sie bei den übrigen $Z$ witterformen so häufig angetroffen wird. Statt dessen sehen wir ein Wesen [Buchfink - Weber ${ }^{1}$, Gimpel - Poll'2)] mit zwei, in der Medianlinie scharf abgegrerizten Körperhälften, von denen jede das ihrer Keimdrüso entsprechende Gepräge trägt, die eine demnach auch äußerlich als männlich, die andere als weiblich in Erscheinung tritt. Auch für diese Zwitter dürfte sich die Annahme bewähren, daß die embryonale Anlage von allem Anfange an geschlechtlich bestimmt sei. Nur wäre sie für solche abnorme Sonderfälle noch durch die, auch von Poll in Erwägung gezogene, Voraussetzung zu ergänzen, daß gleich bei den ersten Furchungsteilungen die Anlagen der beiden Körperhälften eine Geschlechtsverschiedenheit erworben haben, so daß sich die eine in männlicher, die anclere in weiblicher Richtung entwickeln mußte. Diese einmal festgelegte Geschlechtsverschiedenheit der beiden Anlagehälften konnte späterhin durch keinerlei hormonale Einflüsse, sei es der Keimdrüsen, der Zwischenzellen oder anderer Faktoren, mehr verwischt werden. Es sind gleichsam zwei heterogene Halbindividuen, ein männlicher und ein weiblicher Hemiorganismus, in einem Halbseitenzwitter vereinigt.

Es liegt sicherlich nahe, die gleiche Auffassung des Halbseitenzwittertums auch der Erklärung gewisser Fülle von Hermaphroditismus lateralis zugrunde zu legen. Manche sonderbare Asymmetrie und "morphologische Korrelation", wie z. B. das Vorhandenssin der Tuba auf der Ovarialseite und ihr Fehlen auf der Hodenseite, dürfte auf diese Weise leichter verständlich werden.

Die Faktoren, welche die geschlechtsspezifische Differenzierung zuwege bringen, sind uns unbekannt. Daß es die Zwischenzellen sein sollen, ist eine unbegründete Annahme. Bevor sie erscheinen, sind längst schon die Gaschlechtszellen da, die zweifellos ein bestimmtes Vorzeichen tragen, das sie nicht erst von den später auftretenden Zivischenzellen erbalten haben können.

Da wir an die geschlechtsprägende Potenz der Zwischenzellen nicht glauben, müssen wir auch den Hermaphroditis inus interstitialis in jeder Form ablehnen. Für die Zwischenzellen der Keimdrüsen hermaphroditischer Individuen läßt sich keine Regel aufstellen. Häufig sind sie zahlreich, in manchen Fällen wieder spärlich, ohne daß sich eine gesetzmäßige Beziehung zum Geschlechtscharakter erkennen ließe. Wenn die Zwischenzellen den primären wie den sekundüren Geschlechts-

1) Zool. Anzeiger. 13. Jahrg. 1890.

2) Sitzungsber. d. Gesellsch. Naturforschender Freunde. Berlin, Jahrg. 1909. Archiv üü Lntwicklungsmechanik Bd, 47. 
charakter bestimmen sollten, wie will man dann die Gegensätzlichkeit von Keimdrüse und Soma bei den Pseudohermaphroditen erklären? Die Anhänger des Hermaphroditismus interstitialis müssen in einem solchen Falle doch heterologe $Z$ wischenzellen voraussetzen. Warum formen aber diese dann nur das Soma und nicht auch die Keimdrüse in ihrem Sinne, da sie angeblich doch dazu befähigt sein sollen, die »indifferente Anlage« nach ihrem Vorzeichen zu gestalten?

So stehen wir der verbreiteten Meinung über die Zwischenzellen zweifelnd und vielfach ablehnend gegenüber. Wie man zu einer einfacheren und unbefangeneren Auffassung gelangen kann, will ich an einem Beispiele zu zeigen versuchen.

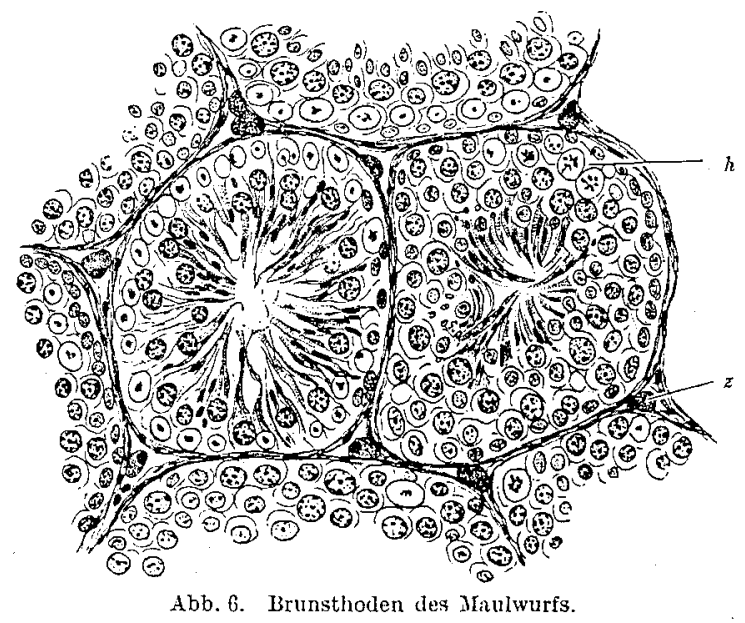

Der Geschlechtscharakter ist, wie schon erwähnt wurde, im allgemeinen bei den Männchen viel bestimmter und stärker ausgeprägt als bei den Weibchen und erreicht in der Brunstzeit seine höchste Entfaltung. Geschlechtstrieb, Angriffslust, Kampfmut sind nun gerade die Eigenschaften, welche angeblich durch die Zwischenzellen angefacht werden und in ihrer Intensität von der Menge der Zwischenzellen abhängig sein sollen ${ }^{x}$ ).

Wie steht es nun um die Zwischenzellen der Brunsthoden?

- Der Hoden vieler Tiere zeigt zu verschiedenen Jahreszeiten ein recht verschiedenes Aussehen. Bekannt sind von Säugetieren insbesondere die Veränderungen des Hodens des Murmeltieres [Hansemann $\left.\left.{ }^{2}\right)\right]$ und des Maulwurfs [Regaud ${ }^{3}$ ), Tandler und Grosz ${ }^{4}$ )].

1) Steinach, Arch. f. Entw.-Mech. 42. Bd. 1916:

2) Virchows Archiv. 142. Bd. 1895.

3) Bibliogr. anatomique. Supplém. 1904.

4) Roux, Arch. f. Entw.-Mech. 33. Bd. 1911. 
In der Begattungszeit -- März, April - sind die Samenkanälchen des Maulwurfhodens auf der Höhe ihrer Entwicklung und strotzend mit Spermatozoen gefültt, die Zwischenzellen aber sehr spärlich. Zu Ende des Frühjahrs beginnen die Samenkanälchen an Durchmesser abzunehmen, die Samenbildung ist eingestellt, die zellige Auskleidung wird vereinfacht. In demselben Maße nehmon aber die Zwischenzellen $\mathrm{zu}$, so daß im Herbste die engen, von einfachem Zylinderepithel ausgekleideten Hodenkanälchen von breiten Zügen lipoidreicher Zwischenzellen getrennt werden. Zur Winterszeit setzt die Vorbereitung zur Spermato-

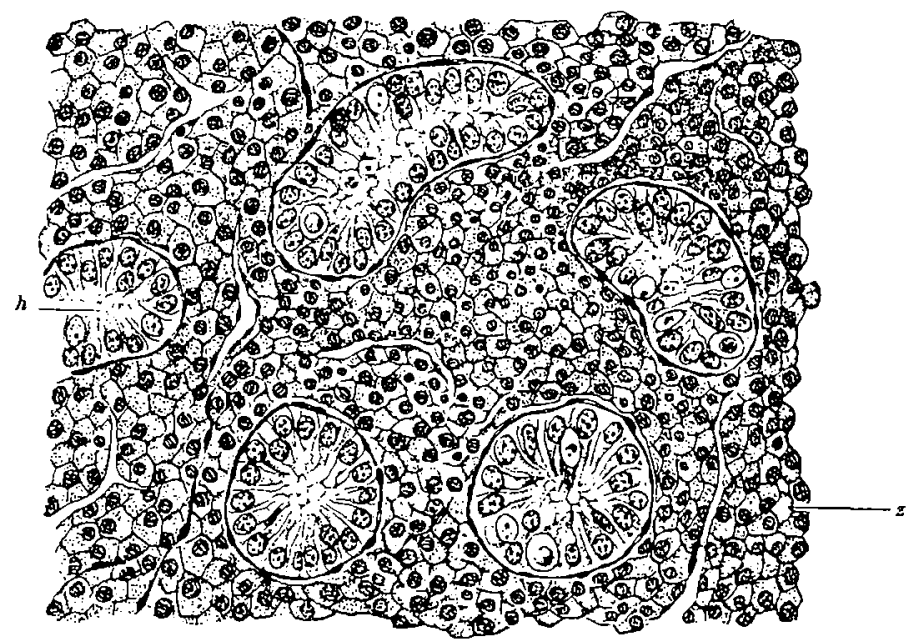

Abb. 7. Hoden des Maulwuris im Ruhestadium.

genese ein. Die Samenzellen vermehren sich reichlich, die Kanälchen werden weiter, und ihre Zellauskleidung wird vielschichtig; aber die Zwischenzellen nelımen mehr und mehr ab und wenn vor Frühjahrsbeginn die Samenbildung in vollem Gange ist, sind sie bis auf geringe Reste verschwunden. Das ist nun aber gerade die Zeit der ungestümen Geschlechtslust, der heftigen Kämpfe um das Weibchen - und die Zwischenzellen haben ihren Tiefstand erreicht, während sie zur Zeit der teilnahmslosen Geschlechtsruhe in ihrer Massenhaftigkeit das Hodenbild beherrschen. Wo bleibt da der Parallelismus von $Z$ wischenzellen und Geschlechtstrieb?

Dagegen wird gerade durch dieses lehrreiche Beispiel eine andere vorurteilslosere Deutung nahegelegt. Die unvoreingenommene Betrachtung des geschilderten Falles macht es wahrscheinlich, daß die Zwischenzellen in ihrer Gesamtheit Bildungen darstellen, welche den Keimdrüsen und vielleicht dem ganzen arterhaltenden Organsystem als trophische Hilfsorgane beigesellt und untergeordnet sind. 
Überlegen wir, was sich am Maulwurfhoden so überzeugend feststellen und an zahIreichen anderen Beispielen, auch an Nichtsäugern [Hoden von Rana esc. - Champy ${ }^{1}$ ), Patzelt ${ }^{2}$ )] leicht bestätigen läßt, so ergibt sich daraus unverkennbar eine gewisse Gegenläufigkeit von generativem und intergenerativem Anteil. Höchstentwicklung der Samenkanälchen fällt mit dem Tiefstand der Zwischenzellen zusammen, und umgekehrt fällt die Höchstentwicklung der Zwischenzellen in eine Zeit, wo die Samenbildung ruht und die Samenkanälchen rückgebildet sind. Sowie die Samenbildung wieder anhebt, nehmen die Zwischenzellen wieder $a b$, gerade so als ob sie aufgezehrt und verbraucht würden, um die wichtige Aufgabe der Samenkanälchen zu ermöglichen, wenn andere Hilfsquellen der Versorgung noch nicht zur Verfügung stehen.

Das dürfte in der Tat auch zutreffen; denn schon im kahlen Winter beginnt sich neues Leben in den Fortpflanzungsorganen vieler Tiere zu regen. $\mathrm{Zu}$ einer Zeit, wo die Ernährungsbedingungen am ungünstigsten sind, muß die Samenbildung - mit ibrem enormen Verbrauch an Nährstoffen für die zahllose Zellvermehrung - durchgeführt werden. Denn im Frühjahr findet die Begattung statt, damit die Jungen in Sommerwärme und Nahrungsfülle heranwachsen, um des Winters Unbilden und Entbehrungen schon genügende Widerstandskraft entgegensetzen zu können.

Im Sommer herrscht in unseren Breiten Überfluß für jung und alt. Da füllen sich die Speicher der Fettorgane, der Winterschlafdrüsen und wahrscheinlich auch die der Zwischenzellen. Für den Winter wird gespart, unnötige Ausgaben werden tunlichst eingeschränkt. So werden auch die anspruchsøollen Samenkanälchen nach erfüllter Aufgabe rückgebildet, wodurch Platz geschaffen wird für die mächtige Entfaltung der Zwischenzellen. Diese scheinen demnach einen lokalen, besonderen Eigenspeicher der Keimdrüsen darzustellen, der im Bedarfsfalle, besonders aber dann, wenn die Spermatogenese und die sonstigen Vorbereitungen für die Fortpflanzung bei behinderter oder erschwerter Nahrungsbeschaffung aus endogenen Mitteln durchgeführt werden müssen, wieder geleert wird, um die zur Erfüllung der Hauptaufgabe des Organismus erforderlichen und geeigneten Stoffe auf kurzem Wege beizustellen.

In Ubereinstimmung mit anderen Autoren (Plato $\left.{ }^{3}\right)$, Lenhossék $\mathbf{k}^{4}$, Kyrles), Patzelto) u. a.] halte auch ich die Zwischenzellen für tro-

1) C. r. Soc. de Biol. 74 .

2) Archiv f. Entw.-Mech. 44. Bd. 1918.

3) Archiv f. mikr. Anat. Bd. 48 u. 50. 1897.

4) Archiv f. Anat. u. Entw. 1897.

5) Sitzungsber. Akad. Wiss. Wien. 120. Bd. 1911.

6) Roux' Archiv f. Entw.-Mech. 44. Bd. 1918. 
phische Bildungen, die man in ihrer Gesamtheit als ein spezifisches Hilfsorgan des arterhaltenden Organsyste ms bezeichnen könnte.

Diese Auffassung weist ihnen einen bescheideneren, aber vielleicht angemesseneren Platz an im Haushalt des Organismus und stellt sie im wesentlichen den Spaicher- oder Vorratsorganen näher als den Drïsen mit innerer Sekretion.

\section{Erklärung der Abbildungen.}

a) Textabbildungen.

Abb. 1. Schematische Darstellung der Keindrüsenanlage im Stadium der Keim. platte. Vereinfacht nach einer Abbildung W. Nagels in Arch. f. mikr. Anat. 34. Bd. Taf. XVIII. Fig. 16: "Querschnitt durch die Eierstocksanlage eines Schweineembryo von $8 \mathrm{~mm}$. $g z$ Geschlechtszelle.

Abb. 2. Schematische Darstellung der Keimdrüsenanlage im Stadium der Sexualstränge. In der Rindensubstanz ( $r i$ ) wie in den Sexualsträngen ( $s$ ) der Marksubstanz eingelagerte Geschlechtszellen.

Abb. 3. Schematische Daritellung der Entwicklung der männlichen Keimdrüse. (Ebenso wie Textabb. 4 nach der Darstellung Winiwarters, Waldeyers und dem Entwurfe Keibels in Keibel -Halls Handbuch d. Entwicklungsgeschichte des Menschen. I. Bd. S. 23.)

$h$ Hodenkanälchen,

$r$ Rete,

$u$ Urogenitalverbindung (Ductuli efferentes),

W Wolffscher Gang,

$Z$ Zwischenzellen.

Abb. 4. Schematische Darstellung der Entwicklung der weiblichen Keimdrüse, wie Textabb. 3 auf Grundlage eines Kintwurfes von Keibel.

$r i$ Rindenschicht mit Follikelbildungen,

$m$ Xrarkstränge (homolog den Hodenkanälchen in Textabb. 3 und gleich diesen Abkömmlinge der Sexualstränge in Textabb. 2).

$Z$ Zwischenzellen,

$r$ Rete,

u Urogenitalverbindung (Epoophoron),

WW Wolffscher Gang.

Abb. 5. Querschnitt durch das Ovarium eines jungen Fuchses (etwa 4 Mronate alt) nach Bühler: Geschlechtsdrüsen der Säugetiere, in O. Hertwigs Handb. d. Entwicklungsiehre. III. Bd. 1906. S. 726.

ri Rindensubstanz,

$m$ Markstränge,

$r$ Rete.

Deutlich die Zweiteilung des Organs in Rinden- und Markschicht. Die Markstränge (gleich den homologen Hodenkanälchen) radiär zum Rete bin verlaufend.

Abb. 6. Aus dem Hoden eines Maulwurfs in der Brunstzeit (März).

$h$ Hodenkanälchen sehr weit, in voller Spermatogenese; die Zwischenräume zwischen den Kanälchen äußerst eng; die Zwischenzellen $(Z)$ spärlich. 
Abb. 7. Aus dem Hoden eines Maulwurfs zur Zeit der Geschlechtsruhe (September).

ih Hodenkanälchen, in Involution, eng, miț einfacher Zellauskleidung; die Zwischenräume zwischen den Kanälchen sehr weit, von zahlreichen Zwischenzellen $(Z)$ ausgefüllt.

Vgl. für Textabb. 6 und 7 die Darstellung Regauds (Comptes rend. de l'Assoc. des Anatomistes. VI. sess. Toulouse 1904. Bibliogr. anat. Supplém. 1904) und die von Tandler und Grosz (Archiv f. Entw.-Mech. Bd. 33. 1911).

\section{b) Abbildungen auf Tafel VI.}

Abb. 1. Durchschnittsbild des Ovariums eines trächtigen Maulwurfs (Talpa europ.). Deutliche Zweiteilung des Organs. Die linke hellere Hälfte ist eigentliches, funktionelles Ovarialgewebe mit typischen Follikelbildungen und einem Corpus luteum $(c l)$; die rechte dunklere Hälfte ist als Testoid $(t)$ aufzufassen, besteht vorwiegend aus Epithelschläuchen, wie sie in $A b b .4$ bei stärkerer Vergrößerung als Markstränge $(m)$ dargestellt wurden, und zeigt am Rande die Kanälchen des Rete $(r)$.

tu Tuba uter., in der Wand der Ovarialtasche eingeschlossen.

Abb. 2. Durchschnitt durch die Zwitterdrüse einer 43jährigen 》Frau «. Fall E. Salén. Nach L. Pick in Arch. f. mikr. Anat. 84. Bd. II. Abt. 1914. Taf. XII. Abb. 7.

$\circ$ Ovarialteil mit Follikelcysten $(f c)$ und einem Corpus luteum $(c l)$,

$h$ Hodenteil mit angedeuteter Septierung und reichlichen Hodenkanälchen.

Die Ähnlichkeit dieser Zwitterdrüse mit den in Abb. 1 und 3 dargestellten Maulwurfsovarien ist unverkennbar und kommt besonders in der Zweiteilung des Organs, in der gleichen Lagerung und dem gleichartigen Aufbau der beiden Organabschnitte deutlieh zum Ausdrnck.

Abb. 3. Ein anderes von der Abb. l einigermaßen verschiedenes Bild des Maulwurfovars aus der Zeit der Geschlechtsruhe. September.

$f$ Follikel im eigentlichen (funktionellen) Ovarialabschnitt,

$t$ Testoid mit MIarksträngen und Zwischenzellen, die in Abb. 5 bei stärkerer Vergrößerung dargestellt sind.

Abb. 4. Randzone des Testoids eines Naulwurfovars in der Tragzeit (entsprechend der Abb. 1).

Dichtgedrängte Markstränge $(m)$, gut ausgebildetes Rete $(r)$.

ep Epoophoronkanälchen.

Abb. 5. Stärker vergrößerte Partie aus dem Testoid der Abb. 3.

$m$ Markstränge, deren ziemlich weite Zwischenräume von zablreichen Zwischenzellen $(Z)$ ausgefüllt werden.

Wer die Abb. 4 und 5 mit den Textabb. 6 und 7 vergleicht, wird sich dem Eindrucke einer weitgehenden Übereinstimmung nicht entziehen können. 